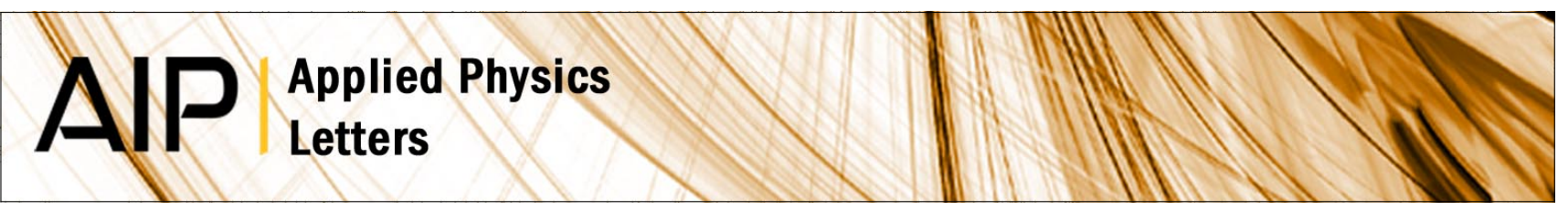

\title{
The role of plasmons and interband transitions in the color of AuAl2, Auln2, and AuGa2
}

V. J. Keast, K. Birt, C. T. Koch, S. Supansomboon, and M. B. Cortie

Citation: Appl. Phys. Lett. 99, 111908 (2011); doi: 10.1063/1.3638061

View online: http://dx.doi.org/10.1063/1.3638061

View Table of Contents: http://apl.aip.org/resource/1/APPLAB/v99/i11

Published by the American Institute of Physics.

\section{Related Articles}

Comprehensive studies of the electronic structure of pristine and potassium doped chrysene investigated by electron energy-loss spectroscopy

J. Chem. Phys. 137, 114508 (2012)

Plasmon resonances and electron transport in linear sodium atomic chains

J. Appl. Phys. 112, 053707 (2012)

Phonon plasmon interaction in ternary group-III-nitrides

Appl. Phys. Lett. 101, 041909 (2012)

Plasmon coupling in circular-hole dimers: From separation- to touching-coupling regimes

J. Appl. Phys. 112, 013113 (2012)

Parameterization of the dielectric function of $\mathrm{InP}$ from 1.19 to $6.57 \mathrm{eV}$ for temperatures from 25 to $700 \mathrm{~K}$

J. Appl. Phys. 112, 013505 (2012)

\section{Additional information on Appl. Phys. Lett.}

Journal Homepage: http://apl.aip.org/

Journal Information: http://apl.aip.org/about/about_the_journal

Top downloads: http://apl.aip.org/features/most_downloaded

Information for Authors: http://apl.aip.org/authors

\section{ADVERTISEMENT}
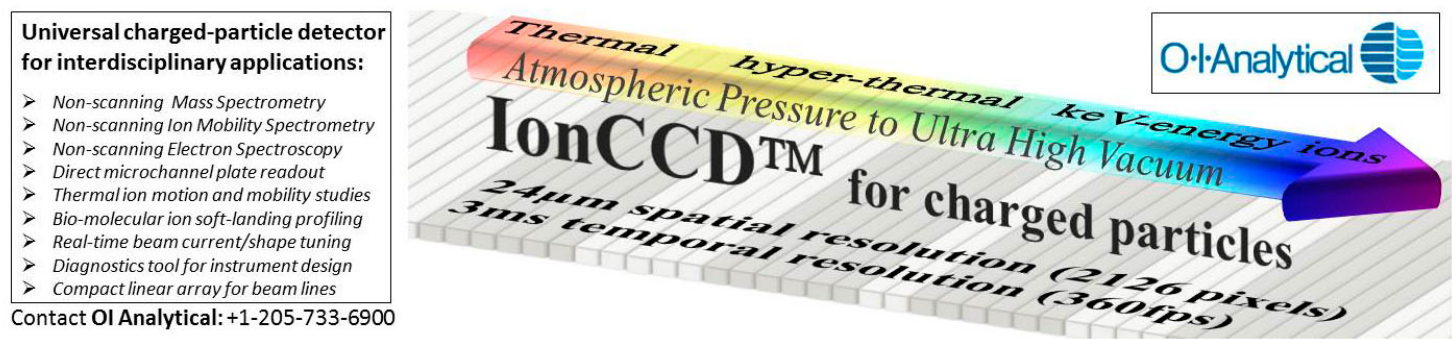


\title{
The role of plasmons and interband transitions in the color of $\mathrm{AuAl}_{2}, \mathrm{AuIn}_{2}$, and $\mathrm{AuGa}_{2}$
}

\author{
V. J. Keast, ${ }^{1, a)}$ K. Birt, ${ }^{1}$ C. T. Koch, ${ }^{2}$ S. Supansomboon, ${ }^{3}$ and M. B. Cortie ${ }^{3}$ \\ ${ }^{1}$ School of Mathematical and Physical Sciences, University of Newcastle, Callaghan NSW 2308, Australia \\ ${ }^{2}$ Max Planck Institute for Metals Research, Stuttgart 70569, Germany \\ ${ }^{3}$ Institute for Nanotechnology, University of Technology, Sydney NSW 2007, Australia
}

(Received 6 December 2010; accepted 24 August 2011; published online 15 September 2011)

\begin{abstract}
First principles calculations of the optical properties of the intermetallic compounds $\mathrm{AuAl}_{2}, \mathrm{AuIn}_{2}$, and $\mathrm{AuGa}_{2}$ have been performed. Analysis of the dielectric functions showed that $\mathrm{AuAl}_{2}$ is unique because a bulk plasmon is seen in the optical region and contributes to the purple color of this material. An experimental electron energy-loss spectrum showed excellent agreement with the theoretical prediction and confirmed the presence of the bulk plasmon. (C) 2011 American Institute of Physics. [doi:10.1063/1.3638061]
\end{abstract}

Intermetallic compounds often have physical properties that are markedly different from those of the constituent elements. The unusual purple and blue colors displayed by $\mathrm{AuAl}_{2}, \mathrm{AuIn}_{2}$, and $\mathrm{AuGa}_{2}$ are an example. Although brittle, these compounds can be used in jewellery ${ }^{1-4}$ and the origin of the color has been discussed in a number of experimental and theoretical investigations. ${ }^{5-13}$

In all three materials the purple and blue hues arise because there is a dip in the reflectivity at around $2 \mathrm{eV}$ which was first measured by Vishnubhatla and Jan in $1967 .{ }^{14}$ This dip has been attributed to the absorption of light due to interband transitions in a similar mechanism to that responsible for the yellow and red colors of gold and copper. Although it has previously been suggested that transitions from the d-band are responsible for the dip, ${ }^{5,9}$ it is now generally accepted that the d-band lies too far below the Fermi-level to be involved..$^{8,10,13,15}$ The assignment of the colors to interband transitions has been made by a qualitative examination of the band structure and/or density of states. An earlier quantitative prediction of the optical response did not provide satisfactory agreement with the experimental data. ${ }^{7}$

We have used density functional theory (DFT) to calculate the electronic structure, optical response, and electron energy-loss spectra (EELS) for these compounds. The theoretical reflectivity has been derived and compared to experimental data. Our calculations suggest that, contrary to the previously accepted view, a plasmon excitation is an important contributor to the optical properties of $\mathrm{AuAl}_{2}$. In order to support this interpretation, experimental EELS were acquired from this material. In EELS, in addition to peaks due to interband transitions, plasmons are directly excited and are also observed as peaks in the spectrum.

A button of $\mathrm{AuAl}_{2}$ of a nominal $2.5 \mathrm{~g}$ mass was produced by melting of the pure elements in a vacuum arc furnace. The sample was formulated to contain a small amount of interdendritic $\mathrm{Al}$ as a second phase, because a $100 \%$ $\mathrm{AuAl}_{2}$ microstructure is exceedingly brittle. The button was then sectioned into slices using a diamond saw. Fig. 1 shows an optical micrograph of the resultant alloy. The sample is

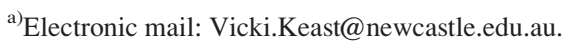

predominantly purple $\mathrm{AuAl}_{2}$ phase with some interdendritic $\mathrm{Al}$ as a minor constituent.

The DFT calculations were performed using the (linearized) augmented plane wave plus local orbitals method $(\mathrm{LAPW}+\mathrm{lo})$ within the wIEN2K software package. ${ }^{16}$ This is an all-electron method that includes relativistic effects. All three alloys have a cubic fluorite structure and belong to space group Fm-3m. The lattice constant used for $\mathrm{AuAl}_{2}$ was $6.00 \AA$, for $\mathrm{AuIn}_{2}$ was $6.502 \AA$, and for $\mathrm{AuGa}_{2}$ was $6.063 \AA .{ }^{17}$ The generalized gradient approximation (GGA) of Perdew, Burke, and Ernzerhof $(\mathrm{PBE})^{18}$ was used for the exchange-correlation potential. The number of $k$ points in the first full Brillouin zone was set to 10000 . Increasing the number of k-points beyond this did not alter spectral details. The complex dielectric function, $\varepsilon=\varepsilon_{1}+i \varepsilon_{2}$, and the loss function, $\operatorname{Im}(-1 / \varepsilon)$, were calculated using the OPTIC routine within wIEN2K. These calculations are based on the random phase approximation and neglect local field effects. ${ }^{19}$ Local field effects are not expected to be significant in the low-energy spectral regions of interest here. $^{20}$

Fig. 2 shows the calculated dielectric functions for the three alloys and Fig. 3 shows the reflectivity spectra derived from these. The calculated reflectivity is compared to experimental data from Vishnubhatla and Jan. ${ }^{14}$ Experimental

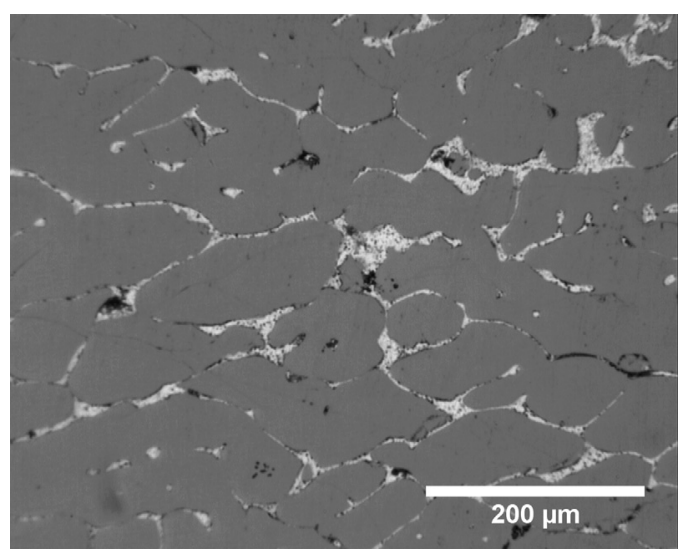

FIG. 1. Optical micrograph of prepared $\mathrm{AuAl}_{2}$ specimen. The major constituent is the purple $\mathrm{AuAl}_{2}$ with a small amount of interdendritic $\mathrm{Al}$ as a second phase. 


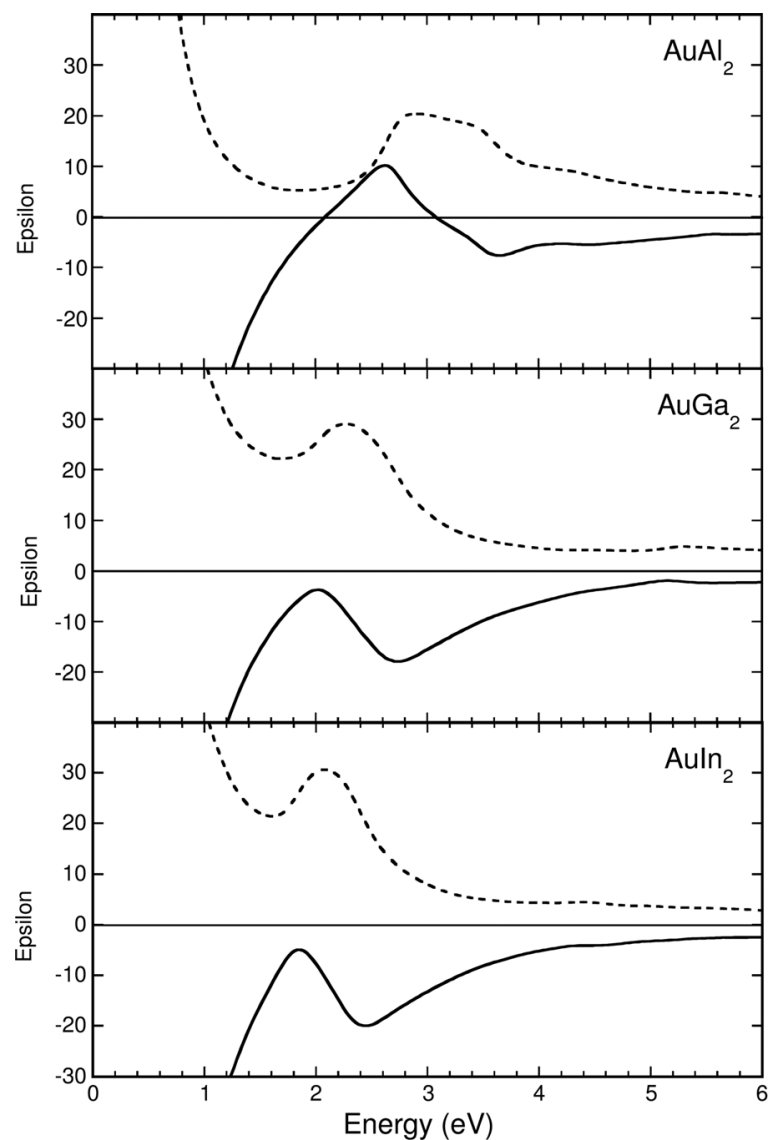

FIG. 2. Real ( $\varepsilon_{1}$, solid curve) and imaginary ( $\varepsilon_{2}$, short-dashed curve) parts of the dielectric functions for gold intermetallic alloys. Calculated using DFT and the RPA.

reflectivity data from our own $\mathrm{AuAl}_{2}$ sample is also included. There will be some contribution from the pure $\mathrm{Al}$ phase to the reflectivity of this sample, but as the reflectivity of $\mathrm{Al}$ in this region is quite featureless and it is a minor constituent, the influence should be minimal.

The theoretical reflectivity spectra were converted into a color for each alloy using the CIELAB color system. ${ }^{21}$ The $\left(\mathrm{L}, \mathrm{a}^{*}, \mathrm{~b}^{*}\right)$ coordinates were as follows: $\mathrm{AuAl}_{2}(64.4,14.5$, -16.7), $\mathrm{AuIn}_{2}\left(87.5, \mathrm{a}^{*}=-3.5,-5.1\right)$, and $\mathrm{AuGa}_{2}$ (84.5, $-1.29,-7.59)$. These color coordinates are all in the bluepurple region with $\mathrm{AuAl}_{2}$ having a more intense and more purple hue than the other two compounds.

Although the reflectivity spectra for these three compounds are quite similar, the dielectric functions reveal a significant difference. Unlike the other two compounds, the $\varepsilon_{1}$ of $\mathrm{AuAl}_{2}$ crosses zero at $2 \mathrm{eV}$. That is, it has a bulk plasma frequency in the optical region. This plasma frequency is significantly modified from the expected position based on a free electron model, as is the case for many similar materials where interband transitions are significant, such as gold and silver. In addition, the onset of interband transitions (the peak in $\varepsilon_{2}$ ) occurs at a higher energy for $\mathrm{AuAl}_{2}$, at $\sim 2.5 \mathrm{eV}$ compared to $\sim 1.8 \mathrm{eV}$ in the other compounds. Therefore the dip in reflectivity for $\mathrm{AuAl}_{2}$, which is responsible for the purple color, cannot be simply attributed to interband transitions. Evidently, the decrease in reflectivity at the plasma frequency that is characteristic of metals plays a key role here too.

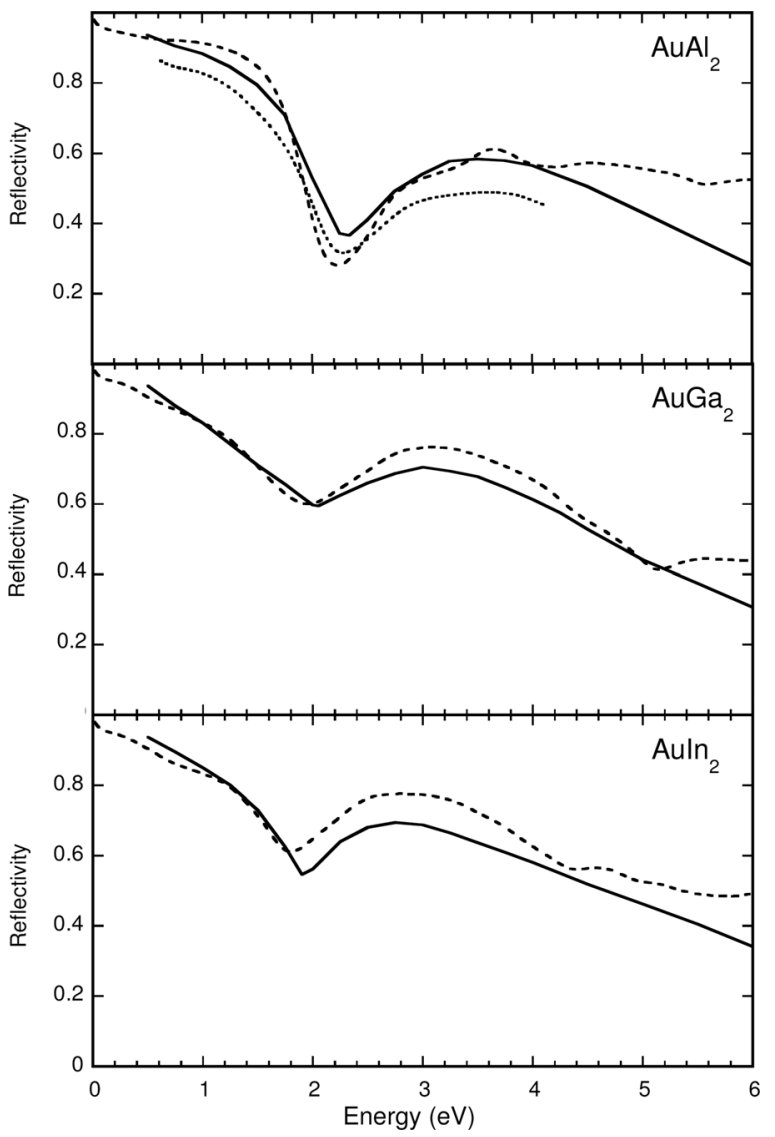

FIG. 3. Calculated (short-dashed curve) and experimental (solid curve) reflectivity (see Ref. 14) for gold intermetallic alloys. Also shown is the experimental reflectivity for $\mathrm{AuAl}_{2}$ measured in this work (dotted curve).

Interestingly, in the first optical study of this alloy, Vishnubhatla and Jan stated that "the possibility of a hybrid plasma-interband resonance in $\mathrm{AuAl}_{2}$, near $2 \mathrm{eV}$, similar to that occurring in beta-brass, cannot be excluded." ${ }^{14}$ They pointed out, however, that the zero crossing of $\varepsilon_{1}$ was highly sensitive to the extrapolation used in the Kramers-Kronig analysis. In the analysis of optical data the narrow energy range of the experimental data mean that energy extrapolations are necessary in order to perform the integrals in the Kramers-Kronig analysis. In the computational results here an extended energy range is available, and this limitation does not apply. It should also be noted that the role of plasmons in the color of beta-brass has still not been conclusively resolved. ${ }^{22}$ Ellipsometry measurements on $\mathrm{AuAl}_{2}$ by Chen and Lynch $^{6}$ also showed the imaginary part of the dielectric function $\left(\varepsilon_{1}\right)$ just crossing zero at around $2.3 \mathrm{eV}$, and the derived energy loss function showed a strong plasmon-like feature around this energy. Despite this, they still attributed the distinctive color entirely to interband transitions. The role of the plasma frequency in the color of this alloy appears to have been largely ignored.

Plasmon resonances can be directly excited by an electron beam and are observed as peaks in the EELS. A thin section of the alloy was prepared using mechanical polishing and ion milling. EELS were acquired using the Zeiss SESAM (sub-electron-volt-sub-angstrom microscope) microscope which incorporates a monochromated electron source, Kohler illumination, and in-column electron spectrometer. ${ }^{23}$ 


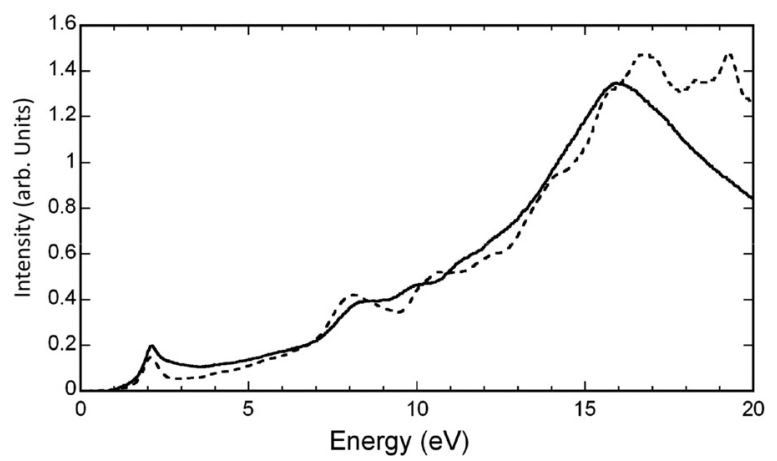

FIG. 4. Calculated (short-dashed curve) and experimental (solid curve) EELS for $\mathrm{AuAl}_{2}$. A bulk plasmon peak is observed at $2 \mathrm{eV}$.

A collection angle of $\sim 4 \mathrm{mrad}$ was used. The spectra were collected from a region approximately $130 \mathrm{~nm}$ in diameter which consisted only of the $\mathrm{AuAl}_{2}$ phase and had a thickness of 1.7 times the inelastic mean free path. The energy resolution, as measured by the full width half maximum of the zerolos peak was $0.17 \mathrm{eV}$. Fig. 4 shows the experimental EELS compared to the theoretical spectra. The vertical axis for the experimental spectra has been rescaled to provide the best visual match. The agreement is striking, and a quite distinct, sharp, peak is observed at $\sim 2 \mathrm{eV}$, where the plasmon peak is expected.

The calculated EELS of $\mathrm{AuIn}_{2}$ and $\mathrm{AuGa}_{2}$ did not display this peak. The calculated density of states (DOS) for the three compounds are not shown here but were consistent with the literature. ${ }^{15,24}$ The DOS for $\mathrm{AuIn}_{2}$ and $\mathrm{AuGa}_{2}$ are almost identical, and $\mathrm{AuAl}_{2}$ has a very similar shape but with slight shifts in the relative energies of features consistent with the higher energy of the interband transitions generating the main peak in $\varepsilon_{2}$. A band analysis of the dielectric function was performed and showed that, as expected, the peak in $\varepsilon_{2}$ is due to transitions between s-p states above and below the Fermi level, and the d-band is not involved.

We have verified here that a low energy bulk plasma frequency is an important contributor to the strong purple color of $\mathrm{AuAl}_{2}$, in contradiction to the common assumption that it is due only to interband transitions. ${ }^{1}$ The occurrence of a metal plasma frequency in the optical region is a quite unusual phenomenon. The bulk plasma frequency is outside the optical region for the otherwise similar materials $\mathrm{AuIn}_{2}$ and $\mathrm{AuGa}_{2}$.

We thank the Advanced Materials Division of Mintek, South Africa for providing the sample of $\mathrm{AuAl}_{2}$ and the Deutscher Akademischer Austaush Dienst (DAAD) for travel support.

${ }^{1}$ R. W. Cahn, Nature 396, 523 (1998).

${ }^{2}$ C. Cretu and E. van der Lingen, Gold Bull. 32, 115 (1999).

${ }^{3}$ J. Fischer-Bühner, A. Basso, and M. Poliero, Gold Bull. 43, 11 (2010).

${ }^{4}$ U. E. Klotz, Gold Bull. 43, 4 (2010).

${ }^{5}$ G. C. Carter, I. D. Weisman, and L. H. Bennett, Phys. Rev. B 5, 3621 (1972).

${ }^{6}$ L. Y. Chen and D. W. Lynch, Phys. Stat. Sol. (b) 148, 387 (1988).

${ }^{7}$ M. B. Cortie, A. Maaroof, G. B. Smith, G. B. Smith, and P. Ngoepe, Current Appl. Phys. 6, 440 (2006).

${ }^{8}$ L. S. Hsu, G. Y. Guo, J. D. Denlinger, and J. W. Allen, J. Phys. Chem. Solids 62, 1047 (2001).

${ }^{9}$ S. Hüfner, J. H. Wernick, and K. W. West, Solid State Comm. 10, 1013 (1972).

${ }^{10}$ I. Perez, B. Qi, G. Liang, F. Lu, M. Croft, and D. Wieliczka, Phys. Rev. B 38, 12233 (1988).

${ }^{11}$ H. R. Philipp, Phys. Stat. Sol. (a) 69, 547 (1982).

${ }^{12}$ K. E. Saeger and J. Rodies, Gold Bull. 10, 10 (1977).

${ }^{13}$ A. C. Switendick and A. Narath, Phys. Rev. Lett. 22, 1423 (1969).

${ }^{14}$ S. S. Vishnubhatla and J. P. Jan, Philos. Mag. 16, 45 (1967).

${ }^{15}$ G. Ugar and F. Soyalp, J. Phys.: Condens. Matter 18, 6777 (2006).

${ }^{16}$ P. Blaha, K. Schwarz, G. K. H. Madsen, D. Kvasnicka, and J. Luitz, WIEN2k, An Augmented Plane Wave + Local Orbitals Program for Calculating Crystal Properties (Karlheinz Schwarz, Techn. Universität Wien, Austria), 2001.

${ }^{17}$ R. W. G. Wyckoff, Crystal Structures (Interscience, New York, 1965).

${ }^{18}$ J. P. Perdew, S. Burke, and M. Ernzerhof, Phys. Rev. Lett. 77, 3865 (1996).

${ }^{19}$ C. Ambrosch-Draxl and J. O. Sofo, Comput. Phys. Commun. 175, 1 (2006).

${ }^{20}$ N. Vast, L. Reining, V. Olevano, P. Schattschneider, and B. Jouffrey, Phys. Rev. Lett. 88, 037601 (2002).

${ }^{21}$ ASTM E308-01, Standard Practice for Computing the Colors of Objects by Using the CIE System (American Society for Testing and Materials, ASTM), 2001.

${ }^{22}$ K. Tarafder, A. Chakrabarti, K. K. Saha, and A. Mookerjee, Phys. Rev. B 74, 144204 (2006).

${ }^{23}$ C. T. Koch, W. Sigle, R. Höschen, M. Rühle, E. Essers, G. Benner, and M. Matijevic, Microsc. Microanal. 12, 506 (2006).

${ }^{24}$ M. A. Miller and G. N. Merrill, J. Phys. Chem. C 112, 6939 (2008). 